Ann. Biol. anim., Bioch., Biophys., Ig66, 6 (2), 2I5-222.

\title{
MISE EN ÉVIDENCE DE DEUX VARIANTS SUPPLÉMENTAIRES DES PROTÉINES DU LAIT DE VACHE : $\alpha_{s 1}-\mathrm{Cn}^{D}$ ET L $g^{D}$
}

\author{
F. GROSCLAUDE, Jeanne PUJOLLE, J. GARNIER \\ et B. RIBADEAU-DUMAS
}

avec la collaboration technique de R. JeLnit, (1) Mireille Favgeras et Monique Veatix

Station centrale de Génétique animale

et Station centrale de Recherches laitières et de Technologie des Produits animaux, Centre national de Recherches sootechniques, 78 -Jouy-en-Josas

\section{SOMMAIRE}

Un type de caséine $\alpha_{s_{1}}$ et un type de $\beta$-lactoglobuline non décrits jusqu'ici ont été observés par analyse électrophorétique en série de laits de vache, de race Flamande pour le premier, Montbéliarde pour le second. L'étude génétique de ces deux types, appelés caséine $\alpha_{s 1} \mathrm{D}$ et $\beta$-lactoglobuline $\mathrm{D}$, montre qu'ils sont déterminés respectivement par un quatrième allèle du locus $\alpha_{s 1}-\mathrm{C} n$ (allèle $\alpha_{s 1}-\mathrm{C} n \mathrm{D}$ ) et par un quatrième allèle du locus $L g$ (allèle $L g \mathrm{D}$ ), dont on donne la fréquence dans les populations considérées.

\section{INTRODUCTION}

La $\beta$-lactoglobuline est la première protéine du lait de vache dont on ait observé - par électrophorèse sur papier à pH 8,6-des variations individuelles (AscHAFFENBURG et DREwRY, I955). Ces auteurs ont montré que les variations qu'ils observaient étaient déterminées d'une manière simple par un locus, appelé $L g$, et pouvant être occupé par les allèles $L g^{\mathbf{A}}$ et $L g^{\mathbf{B}}$ (Aschaffendurg et DrEwRY, I957 u). II s'agit d'un locus de structure autosomal, responsable de la synthèse des deux types ou "variants" de la protéine, qui sont co-dominants.

(1) Station expérimestale laitière, 39 - P'oligny. 
BELI, (I962), utilisant l'électrophorèse en gel d'amidon à $\mathrm{pH} 8,6$, a décrit une troisième forme de $\beta$-lactoglobuline appelée $\mathrm{C}$ (non distincte de $\mathrm{B}$ en électrophorèse sur papier à $\mathrm{pH} 8,6$ ), et synthétisée par un troisième gène allèle des deux précédents (allèle $L g^{\mathrm{C}}$ ).

De la même façon, l'électrophorèse - sur papier, en gel d'acrylamide ou en gel d'amidon - a révélé l'existence de variations héréditaires des caséines $\alpha_{81}$, $\beta$ et $x$ du lait de vache. L'analyse génétique de ces variations a permis de mettre en évidence les trois loci de structure autosomaux $\alpha_{s 1}-C n, \beta-C n$ et $x-C n$ qui contrôlent la synthèse de ces trois espèces de caséines : le locus $\beta-C n$ peut être occupé par les gènes allèles $\beta-C n^{\mathrm{A}}, \beta-C n^{\mathrm{B}}, \beta-C n^{\circ}$, responsables de la synthèse des variants de caséine $\beta \mathrm{A}, \mathrm{B}$ et $\mathrm{C}$ (ASCHAFFENBuRG, I96I, I963) ; le locus $\alpha_{s 1}-C n$ peut être occupé par les allèles $\alpha_{s 1}-C n^{\mathrm{A}}, \alpha_{s 1}-C n^{\mathrm{B}}$ et $\alpha_{s 1^{-}} C n^{0}$ synthétisant les variants de caséine $\alpha_{s 1} \mathrm{~A}$, $\mathrm{B}$ et $\mathrm{C}$ (Thompson et al., Ig62; KIDDy et al., I964); enfin le locus $x$ - $C n$ peut être occupé par les allèles $x-C n^{\mathrm{A}}$ et $x-C n^{\mathrm{B}}$, synthétisant les variants de caséine $x \mathrm{~A}$ et $\mathrm{B}$ (Schmidt, I964; NeElin, I964; WoychiK, I965). Ces trois loci de structure sont par ailleurs étroitement liés (GROSCLAUDE et al., I964, I965; KING et al., I965).

Les variants des caséines et de la $\beta$-lactoglobuline décrits à l'étranger ont été trouvés dans plusieurs races françaises à 1'exception du type $\mathrm{A}$ de caséine $\alpha_{s}$, et du type $\mathrm{C}$ de $\beta$-lactoglobuline ; par contre, nous avons observé deux types supplémentaires : un type de caséine $\alpha_{s 1}$, appelé $\mathrm{D}$ et un type de $\beta$-lactoglobuline également appelé D. Nous allons présenter successivement ces deux " nouveaux » variants.

\section{RÉSULTATS}

\section{Le type $D$ de caséine $\alpha_{s}$}

\section{a) Technique et observations initiales.}

Dans le cadre d'études systématiques sur les variations héréditaires des protéines du lait de vache, entreprises dans les races françaises, nous avons analysé, par électrophorèse en gel de polyacrylamide, selon la technique d'AschafFENBURG (I963), le lait de 380 vaches de race Flamande. Ces analyses, effectuées en novembre et décembre 1964 , ont révélé l'existence, dans la zone des caséines $\alpha_{s 1}$, d'une bande protéique inconnue jusqu'ici. Cette bande, s'observant entre les bandes $\mathrm{A}$ et $\mathrm{B}$ et sensiblement à égale distance de celles-ci, a été rencontrée chez 29 individus différents,
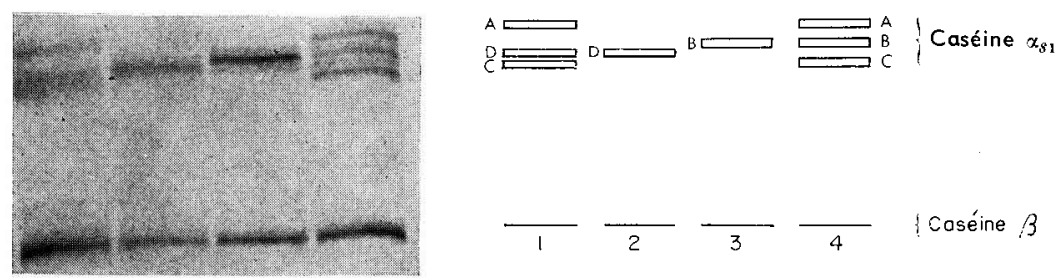

FIs. I. - Mobilité relative des bandes de caséine $\alpha_{s 1}$ en gel d'amidon avec urée (WAKE et BALDWIN, I96I) et mercapliéthanol

$\mathbf{I}=$ mélange d'une caséine $\alpha_{s 1}-\mathrm{D}$ et d'une caséine $\alpha_{\delta 1}-\mathrm{A} / \mathrm{C}$;

$2=$ caséine $\alpha_{s 1}-\mathrm{D} ; 3=$ caséine $\alpha_{s 1}-\mathrm{B} ; 4=$ mélange d'une caséine $\alpha_{s 1}-\mathrm{B}$ et d'une caséine $\alpha_{s 1}-\mathrm{A} / \mathrm{C}$ 
issus de 21 des 56 élevages visités. Dans 25 cas elle était associée à la bande $B$, dans deux cas à la bande C, et dans deux cas on l'observait seule, avec une intensité plus forte que dans les cas précédents (fig. I). Ces résultats suggéraient l'existence, au locus $\alpha_{s 1}$-Cn, d'un quatrième allèle $\alpha_{s 1}$-Cn $\mathrm{D}$.

Quelques mois plus tard, de nouveaux échantillons de 24 de ces laits ont été analysés par élec. trophorèse en gel de polyacrylamide selon une autre technique (PEterson, 1963). Les résultats ont été identiques. Pour certains individus, les deux analyses successives intervenaient à des stades différents d'une même lactation; pour les autres, elles se plaçaient respectivement à la fin et au début de deux lactations consécutives. Nos observations étaient donc reproductibles.

\section{b) Déterminisme génétique.}

Pour vérifier que le type $\mathrm{D}$ est le produit d'un allèle du locus $\alpha_{s 1^{-}} C n$, nous avons recherché des familles permettant d'effectueí un test d'allélisme. Rappelons que, de manière générale, effectuer un test d'allélisme, c'est vérifier qu'un individu possédant deux gènes présumés allèles transmet toujours 1'un de ces deux gènes à ses descendants, mais jamais les deux à la fois. Par ailleurs, s'il existe un allèle $\alpha_{s 1}-C n^{\mathrm{D}}$, on doit trouver - d'autant plus souvent que sa fréquence est plus élevée - des individus homozygotes, qui ne produisent que le type D.

Notre hypothèse nous semble confirmée par les observations suivantes :

- le fait que, dans deux cas, la bande D ait été rencontrée seule et plus intense, suggère, par analogie avec ce qui a été observé jusqu'ici pour les caséines $\alpha_{s 1}$ et $\beta$ bovines, que les deux individus porteurs sont homozygotes pour la bande $\mathrm{D}$, donc de formule génotypique $\alpha_{s 1}-C n^{n} /-\alpha_{s 1} C n^{n}$. Malheureusement aucune de ces deux vaches n'a de fille en lactation;

- une des vaches présumées homozygotes était fille d'un taureau, Brillant de $l^{\prime} Y s e r$, dont une autre fille était $\alpha_{s 1}-C n^{\mathrm{B}} / \alpha_{s 1}-C n^{\mathrm{B}}$. Ce taureau était donc hétérozygote,

TABLEAU I

Répartition des phénotypes et des génotypes correspondants dans la population borine flamande de Flandre maritime

\begin{tabular}{|c|c|c|c|}
\hline Caséines $\alpha_{s 1} \quad$ Caséines $\beta$ & $A=\beta-C n^{A} / \beta-C n^{A}$ & $\frac{\mathrm{AB}}{\beta-\mathrm{C} n^{\mathrm{A}} / \beta-\mathrm{C} n^{\mathrm{B}}}$ & $\mathrm{B}=\beta-\mathrm{C} n^{\mathrm{B}} / \beta-\mathrm{C} n^{\mathrm{B}}$ \\
\hline $\mathrm{B}=\alpha_{s 1}-C n^{\mathrm{B}} / \alpha_{s 1}-C n^{\mathrm{B}}$ & 245 & 12 & 1 \\
\hline $\mathrm{BC}=\alpha_{s 1}-C n^{\mathrm{B}} / \alpha_{s 1}-C n^{\mathrm{C}} \ldots \ldots \ldots$ & 56 & 3 & - \\
\hline $\mathrm{C}=\alpha_{s 1}-C n^{\mathrm{C}} / \alpha_{s 1} \cdot C n^{\mathrm{C}} \ldots$ & 4 & 一 & - \\
\hline $\mathrm{D}=\alpha_{s 1}-C n^{\mathrm{D}} / \alpha_{s 1}-C n^{\mathrm{D}} \ldots \ldots \ldots$ & 2 & - - & 一 \\
\hline $\mathrm{BD}=\alpha_{s 1}-C n^{\mathrm{B}} / \alpha_{s 1}-C n^{\mathrm{D}} \ldots \ldots$ & 24 & 1 & 一 \\
\hline $\mathrm{CD}=\alpha_{s 1}-C n^{\mathrm{C}} / \alpha_{s 1}-C n^{\mathrm{D}}$ & 2 & - & 一 \\
\hline
\end{tabular}

de formule génotypique $\alpha_{s 1}-C n^{\mathrm{B}} / \alpha_{s 1}-C n^{\mathrm{D}}$. L,e lait de trois autres de ses filles, ainsi que celui de leurs mères a pu être analysé. Deux de ces filles avaient reçu $\alpha_{s 1}-C n^{\mathrm{D}}$ de leur père, l'autre $\alpha_{s 1}-C n^{\mathrm{B}}$. 
Par ailleurs, quatre autres vaches de type BD ont transmis deux fois la bande $\mathrm{D}$ à leur fille et deux fois la bande $\mathrm{B}$. Ces animaux avaient donc vraisemblablement le génotype $\alpha_{s 1}-C n^{\mathrm{B}} / \alpha_{s 1}-C n^{\mathrm{D}}$.

Les résultats complets d'analyse des 380 échantillons individuels sont donnés dans le tableau I, en admettant l'existence d'un quatrième allèle $\alpha_{s 1}-C n^{\mathrm{D}}$. En supposant, ce qui paraît vraisemblable, que les combinaisons $\alpha_{s 1^{-}} C n^{\mathrm{C}}-\beta-C n^{\mathrm{B}}$ et $\alpha_{s 1}-C n^{\mathrm{D}}-\beta-C n^{\mathrm{B}}$ n'existent pas dans 1'échantillon, les estimations des fréquences des combinaisons $\alpha_{s 1}-C n-\beta-C n$ dans la population bovine Flamande, obtenues par simple comptage, sont les suivantes :

$$
\begin{array}{lll}
\alpha_{81}-C n^{\mathrm{B}}-\beta-C n^{\mathrm{A}} & : 0,81 \pm 0,04 \\
\alpha_{s 1}-C n^{\mathrm{c}}-\beta-C n^{\mathrm{A}}: & 0,09 \pm 0,02 \\
\alpha_{81}-C n^{\mathrm{B}}-\beta-C n^{\mathrm{B}}: & 0,06 \pm 0,02 \\
\alpha_{s 1}-C n^{\mathrm{D}}-\beta-C n^{\mathrm{\Lambda}}: & 0,04 \pm 0,02
\end{array}
$$

Le nombre observé de 2 homozygotes correspond sensiblement au nombre attendu dans l'hypothèse d'équilibre génétique ( $\mathrm{I}$ à 2), ce qui confirme encore 1'interprétation donnée à nos observations.

c) Mobilité relative du type $D$; discussion.

Nous avons cherché à définir la mobilité relative du type $\mathrm{D}$ selon le principe proposé par WAKE et BALDWIN (I96I) et adopté par Thompson et al. (I964, I965), en opérant en gel d'acrylamide car on obtient, avec ce gel, une meilleure séparation des caséines $\alpha_{s 1}$ qu'avec le gel d'amidon. Nous disposions, pour ce travail, d'une cellule à électrophorèse verticale identique à celle de THompson et al. (cellule construite par E. C. Apparatus Company), et d'échantillons de caséines $\alpha_{s 1} \mathrm{~A}, \mathrm{~B}$ et C fournis par ces auteurs.

D’une manière générale, nous avons constaté qu'il fallait respecter strictement le protocole de Thompson et al. (I964) pour obtenir des indices de mobilité relative voisins des indices donnés par ces auteurs (respectivement I,22, I, I3 à I, I4 et I, Io pour les types A, B et C). En modifiant ce protocole, par exemple en effectuant la "prémigration" ( $\left.{ }^{1}\right)$ de deux heures recommandée par le constructeur de la cellule d'électrophorèse, on peut altérer franchement l'échelle des mobilités.

De toutes façons, en opérant dans un même laboratoire et dans des conditions apparemment constantes, les valeurs des indices de mobilité peuvent varier de 1'ordre de \pm o,or (Thompson, I966) ; aussi peut-on s'attendre à de légers écarts entre les valeurs obtenues par des laboratoires différents. En ce qui concerne la caséine $\alpha_{s 1} \mathrm{D}$, nous avons observé la valeur de I, I5, mais ceci dans une échelled e mobilités un peu plus lente que celle de Thompson et al. (rg64) (respectivement I,2I, I,I2 et I,og pour les types A, B et C). Il se trouve que Thompson (I966) analysant un échantillon de notre caséine obtient également la valeur de I, I5, mais dans une échelle plus proche de la nôtre que de son échelle initiale. Dans cette dernière, $\alpha_{s 1}-C n^{\mathrm{D}}$ aurait vraisemblablement un indice de mobilité de r, r6.

Compte tenu de ces observations, il nous semble, en conclusion, qu'il serait préférable, pour identifier un variant, de pouvoir disposer d'échantillons de référence de tous les variants déjà connus.

(1) La is prémigration a consiste à faire passer le courant électrique dans le gel prcalablement à l'insertion des échantillons. 


\section{Le type $D$ de $\beta$-lactoglobuline}

\section{a) Technique et observations initiales.}

Le type D de $\beta$-lactoglobuline a été découvert au cours de l'analyse de 762 échantillons de lait provenant de 364 mères et de leurs 398 filles, récoltés à l'origine pour l'étude des caséines $\varkappa$. Les analyses ont été faites sur les lactosérums recueillis après précipitation des caséines à $\mathrm{pH}$ 4,6 par HCl normal. Ayant constaté que la technique d'analyse électrophorétique des caséines [technique de WAKE et BALDWIN (I96r) avec addition au gel de mercaptoéthanol] se prêtait avantageusement à la détermination des types de $\beta$-lactoglobuline, nous avons appliqué cette technique, pour des raisons de commodité, à l'analyse en série des 762 échantillons de lactosérum.

Dans ces conditions, en lisant les électrophorégrammes de l'anode vers la cathode (ordre des vitesses de migration décroissantes) on rencontre successivement la bande $d^{\prime} \alpha$-lactalbumine, puis les bandes de $\beta$-lactoglobulines A, B et D) (fig. 2). Les $\beta$-lactoglobulines B et C ne sont pas séparées l'une de l'autre.

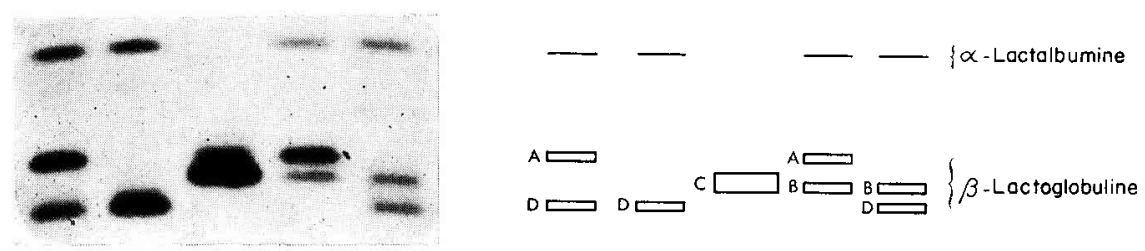

FIG. 2. - Mobilité relative des bandes de $\beta$-lactoglobuline bovine en gel d'amidon avec urée (WAKE et BALDWIN, I96I) et mercaptoéthanol

Les bandes $\mathrm{C}$ et $\mathrm{B}$ ont, dans ce gel, la même mobilité La bande C est ici épaissie par un excès de protéine.

La forme $\mathrm{D}$ a été rencontrée soit associée à $\mathrm{A}$ ou $\mathrm{B}$, soit seule (r vache), l'intensité des bandes correspondant respectivement dans ces deux cas à celle des bandes observables chez des individus reconnus hétérozygotes ou homozygotes pour les variants décrits jusqu'ici.

\section{b) Déterminisme génétique.}

Notre échantillon de vaches Montbéliardes était constitué de couples mèrefille. Les pères de ces filles, étant des taureaux d'insémination artificielle, ont une nombreuse descendance, et nous avons pt1 déterminer leurs génotypes au locus $L g$ en observant précisément dans l'échantillon les variants qu'ils transmettaient à leurs filles.

Remarquons que lorsqu'un taureau transmet alternativement deux variants différents, on a la certitude qu'il est hétérozygote; par contre, s'il ne transmet jamais qu'un seul variant, on ne peut le dire homozygote qu'avec une certaine vraisemblance, d'autant plus grande qu'on aura étudié un plus grand nombre de ses filles.

Toutes les familles comptant au moins un représentant porteur du type D figurent dans le tableau 2 où nous avons utilisé une écriture simplifiée pour les génotypes paternels (A/B au lieu de $L g^{\mathrm{A}} / L g^{\mathrm{B}}$ par exemple).

La fréquence de 1'allèle $L g^{\mathrm{D}}$ dans la race $M$ ontbéliarde, estimée sur l'échantillon des 364 mères, est de $0,02 \pm 0,0$ I alors que $L g^{\mathrm{A}}$ et $L g^{\mathrm{B}}$ ont respectivement pour fréquence $0,52 \pm 0,04$ et $0,46 \pm 0,04$. La probabilité de rencontrer dans notre échantillon une vache homozygote pour $L g^{\mathrm{D}}$, dans l'hypothèse d'équilibre génétique de 
la population, est faible (de l'ordre de 0,12 ). L'utilisation en insémination artificielle de deux taureaux porteurs de cet allèle explique que nous en ayons cependant rencontré une qui est fille de l'un de ces taureaux.

TABLEAU 2

Analyse des fanilles dont l'un des représentants au moins possède la $\beta$-lactoglobuline $D$

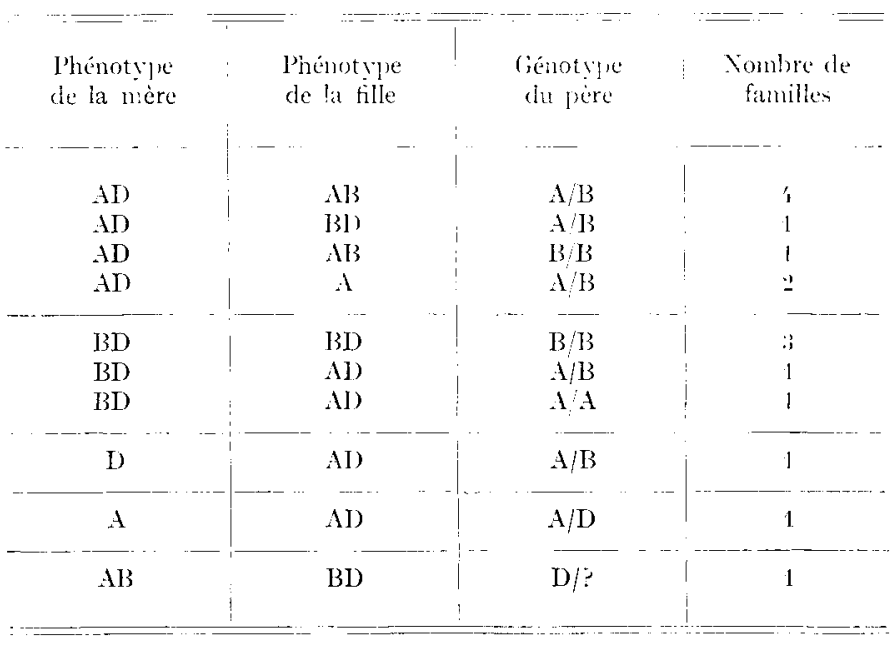

c) Mobilités relatives des quatre variants; discussion.

Les formes $\mathrm{A}, \mathrm{B}, \mathrm{C}$ et $\mathrm{D}$ ont été comparées à partir de préparations pures de $\beta$-lactoglobuline; la forme $D$, en particulier, a été obtenue à l'état cristallisé, selon la technique d'AschafFenburg et DREWRy ( 1957 b) en partant du lait de notre seule vache homozygote. Plusieurs techniques d'électrophorèse ont été mises en cuvre et ont donné les résultats suivants :

- en gel de polyacrylamide et tampon véronal à pH 8,6 (Aschafrenburg, I964), A, B et D ont les mêmes mobilités relatives qu'en gel d'amidon avec urée et mercaptoéthanol, mais $C$ se sépare inégalement de $B$, dont elle est parfois très voisine, parfois bien distincte;

- en gel d'amidon à $\mathrm{pH} 8,6$, dans des conditions apparemment identiques à celles utilisées par BELL (I962), les bandes B et $\mathrm{C}$ sont confondues, alors que $\mathrm{A}$, $B$ et $\mathrm{D}$ ont toujours les mêmes mobilités relatives;

- en gel de polyacrylamide (concentration de 5 p. I0o) et tampon phosphate à $\mathrm{pH} 6,8$, les quatre variants se séparent bien et s'échelonnent dans l'ordre $\mathrm{A}, \mathrm{B}$, $\mathrm{C}$ et $\mathrm{D}$, avec des espacements presque égaux ;

- enfin, en gel d'agar, les bandes $C$ et $D$ migrent plus lentement que $B$, mais se distinguent mal l'une de l'autre, alors que sur papier, en tampon véronal à $\mathrm{pH} 8,6$, $\mathrm{D}$, contrairement à $\mathrm{C}$, se sépare bien de B. (Aschaffenburg, I965).

La détermination de la composition en acides aminés de la $\beta$-lactoglobuline D est en cours à Jouy-en-Josas. La connaissance complète des substitutions de résidus 
entre les quatre formes $\mathrm{A}, \mathrm{B}, \mathrm{C}$ et $\mathrm{D}$ permettra de donner une explication aux observations faites sur leurs mobilités relatives. Il semble néanmoins, dès à présent, que le comportement du type C qui ne se sépare bien de $B$ en gel d'acrylamide qu'en abaissant le $\mathrm{pH}$ à 6,8 , puisse s'expliquer par la substitution d'une histidine à une glutamine qui différencie le type $\mathrm{C}$ du type $\mathrm{B}$ (KALAN et al., I964). En effet, le résidu d'histidine n'est pas protoné, normalement à $\mathrm{pH} 8,6$. Nous avons relevé le cas de mutations de l'hémoglobine humaine, impliquant un résidu d'histidine, et qui se traduisent par un comportement électrophorétique analogue, expliqué de la même façon, par exemple celui de l'hémoglobine "Zürich » (MUI,LER et KINGMA, I96r).

Notons enfin que l'on n'obtient pas, en gel d'amidon avec urée sans mercaptoéthanol, une aussi bonne résolution des bandes de $\beta$-lactoglobuline que dans le même gel en présence de mercaptoéthanol, ce qui peut s'expliquer ainsi : l'urée provoquant le déploiement des chaînes de $\beta$-lactoglobuline, extériorise les groupements sulfhydryles et permet alors la formation de ponts disulfure entre chaînes, et d'agrégats responsables de la mauvaise résolution électrophorétique ; le mercaptoéthanol, empêchant la formation de ces ponts disulfure, permet d'effectuer le tri électrophorétique sur les chaînes élémentaires, ce qui correspond aux conditions les plus souhaitables dans l'étude d'un polymorphisme d'origine génétique. CHERNOFF et PETTTI (r964) ont observé un effet analogue du mercaptoéthanol lors de la séparation électrophorétique des chaînes de l'hémoglobine humaine, et l'ont expliqué d'une manière similaire.

\section{NOTE}

Un type de $\beta$-lactoglobuline apparemment identique au type I) (ASCHAFFENBURG, I965) a été observé indépendamment par MEYER (I965) dans les races allemandes Höhenfleckvieh et Brawnvieh.

$$
\text { Recu pour publication an firrier } \mathrm{x} 966 .
$$

\section{REMERCIEMENTS}

Nous remercions le Dr M. P. Thompson et le Dr li. B. Kalan qui nous ont foumi des échantillons de référence de caséine et de $\beta$-lactoglobuline. Jous sommes reconnaissants à M. L. Pr.LEREIN d'avoir bien voulu effectuer l'ensemble des prélevements de lait dans la race Flamande.

\section{SUMMARY}

ADDITIONAL VARIANTS OF $\alpha_{s 1}$ - CASEIN AND $\beta$-LACTUGLOBULIN IN CATTLE

Two additional electrophoretic variants of cattle milk proteins are described : a variant of $\alpha_{s_{1}}$-casein, found in the Flamande breed and called $\alpha_{s_{1}}$-casein $\mathrm{J}$, and a variant of $\beta$-lactoglobulin, found in the Montbeliarde breed, and called $\beta$-lactoglobulin D (fig. I and 2). These variants are shown to be determined by a fourth allele of the $\alpha_{s 1}-\left(n_{n}\right.$ locus (allele $\alpha_{11}-C n^{\mathrm{D}}$ ), and by a fourth allele of $L g$ locus (allele $L g^{1}$ ) respectively. 'The frequency of $\alpha_{s 1}-C n^{\mathrm{D}}$ in the Filanande breed is $0,04: 0,02$, and that of the $L g^{\mathrm{D}}$ in the Montbéliarde breed is about $0,02 \cdots 0,01$. Relative clectrophoretic mobilities of these variants are studied and discussed. 


\section{RÉFÉRENCES BIBLIOGRAPHIQUES}

AschafFenblRg; R., I96r. Inherited casein variants in cow's milk. Nature, 192, 43 I.

ASCHAFFENBURG R., 1963. Inherited casein variants in cow's milk. II. Breed differences in the occurence of 3 -casein variants. J. Dairy Res., 30, $25^{\mathrm{I}-258}$.

Aschaffenburg R., 1964. Protein phenotyping by direct polyacrylamide-gel electrophoresis of whole milk. Biochen, Biophys. Acta, 82, i 88-r91.

Ascimaffenburg R., 1965. (Communications personnelles).

Aschaffenburg R., Drewry J., r955. Occurence of different $\beta$-lactoglobulins in cow's milk. Vature, 176, 2 I 8-2I9.

Aschaffenburg R., Drewry J., ig57 a. Genetics of the $\beta$-lactoglobulins of cow's milk. Valure, 180, $376-378$.

Aschaffenblrg R., Drewry J., $1957 b$. Improved method for the preparation of crystalline $\beta$-lactoglobulin and $\alpha$-lactalbumin from cow's milk. Biochem. J., 65, 273-277.

BeLL K., 1962. One dimensional starch gel electrophoresis of bovine skim milk. Nature, 195, 705-706.

Chernoff A. I., Pettit N. M., 1964. The amino-acid composition of hemoglobin. III. A qualitative method for identifying abnormalities of the polypeptide chains of hemoglobin. Blood, 24, 750-756.

Grosclaude F., Garnier J., Ribadeau-Dumas B., Jeunet R.,, ig64. Étroite dépendance des loci contrôlant le polymorphisme des caséines $\alpha_{s}$ et $\beta$. C. R. Acad. Sci., 259, I 569-1 571 .

Groscladde F., Pujolle Jeanne, Garnier J., Ribadeau-Dumas B., ig65. Déterminisme génétique des caséines $x$ du lait de vache ; étroite liaison du locus $x$-Cn avec les loci $\alpha_{s}-C n$ et $\beta-C n$. C.R. Acad. Sci., 261, 5229-5232.

Kalan E. B., Greenberg R., Watter M., Gordon W. G., r964. Chemical properties of $\beta$-lactoglobulins A, B et C. Biochem. Biophys. Res. Commun., 16, I99-203.

Kiddy C. A., Johnston J. O., Thompson M. P.,.I964. Genetic polymorphism in casein variation. J. Dairy Sci., 47, I47-I 5I.

King J. W. B., Aschaffenburg R., Kiuby C. A., Thompson H. P'., ig65. Non-independent occurence of $\alpha_{s}$ and $\beta$-casein variants of cow's milk. Nature, $206,424$.

Meyer H., 1965. (Communication persomelle).

Muller C. J., Kingma S., I961. Haemoglobin Zürich : $\alpha_{\mathbf{2}}{ }^{\mathrm{A}} \beta_{\mathbf{2}}{ }^{\mathbf{6 3}}$ Arg. Biochim. Biophys. Acta, 50, 595 .

NeELin J. H., 1964. Variants of $x$-casein revealed by improved starch gel electrophoresis. J. Dairy Sci., 47, 506-509.

Peterson R. F., I 963 . High resolution of milk proteins obtained by gel electrophoresis. J. Dairy S:i., 45, I I 36 - I 139 .

Sснмпт D. C., 1964. Starch gel electrophoresis of $x$-casein. Biochim. Biophys. Acla, 90, 41 I-4I4.

Thompsos M. P., ig66. (Communication personnelle).

Thompson M. P., Kiddy C. A., Johnston J. O., Weinberg R. M., I964. Genetic polymorphism in caseins of cow's milk. II. Confirmation of the genetic control of $\beta$-casein variation. J. Dairy Sci., 47, 378$38 \mathrm{r}$.

Thompson M. P., Kiddy C. A., Pepper L., ZitTle C. A., I962. Variations in the $\alpha_{8}$-casein fraction of individual cow's milk. Nature, 195, 100I-1002.

Thompson M. P., Tarassuk N. P., Jenness R., Lillevik H. A., Asirwortit U. S., Rose D., r965. Nomenclature of the proteins of cow's milk. Second revision. J. Dairy Sci., 58, 159-169.

WAKE R. C., BALDWiN R. L., ig6r. Analysis of casein fraction by zone electrophoresis in concentrated urea. Biochim. Biophys. Acta, 47, 225-239.

Woychiк J. II., Ig64. Polymorphism in $x$-casein of cow's milk. Biochem. Biophys. Research Commun., 16, $267-271$.

Woycilik J. H., 1965. Phenotyping of $x$-casein. J. Dairy Sci., 48, 496-497. 